\title{
Article
}

\section{Challenges to clinical pharmacy practice in Nigerian hospitals: a qualitative exploration of stakeholders' views}

\author{
Auta, Asa, Maz, Julia and Strickland-Hodge, Barry \\ Available at http://clok.uclan.ac.uk/14190/ \\ Auta, Asa ORCID: 0000-0001-6515-5802, Maz, Julia and Strickland-Hodge, \\ Barry (2016) Challenges to clinical pharmacy practice in Nigerian hospitals: a \\ qualitative exploration of stakeholders' views. Journal Of Evaluation In Clinical \\ Practice. ISSN 1356-1294
}

It is advisable to refer to the publisher's version if you intend to cite from the work. http://dx.doi.org/10.1111/jep.12520

For more information about UCLan's research in this area go to http://www.uclan.ac.uk/researchgroups/ and search for < name of research Group>.

For information about Research generally at UCLan please go to http://www.uclan.ac.uk/research/

All outputs in CLoK are protected by Intellectual Property Rights law, including Copyright law. Copyright, IPR and Moral Rights for the works on this site are retained by the individual authors and/or other copyright owners. Terms and conditions for use of this material are defined in the policies page.

\section{CLoK}

Central Lancashire online Knowledge www.clok.uclan.ac.uk

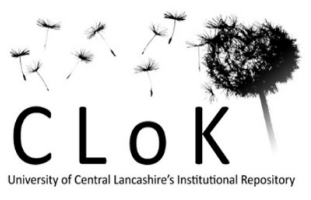


Challenges to clinical pharmacy practice in Nigerian hospitals: a qualitative exploration of stakeholders' views

Asa Auta, MPH

PhD Candidate, School of Healthcare, Faculty of Medicine and Health, University of Leeds, Leeds, LS2 9JT, UK.

Barry Strickland-Hodge, PhD, FRPharmS

Senior Pharmacy Lecturer, School of Healthcare, Faculty of Medicine and Health, University of Leeds, Leeds, LS2 9JT, UK.

Julia Maz, PhD

Lecturer, School of Healthcare, Faculty of Medicine and Health, University of Leeds, Leeds, LS2 9JT, UK.

Corresponding author: Asa Auta, School of Healthcare, Faculty of Medicine and Health, University of Leeds, Leeds, LS2 9JT, UK. Email: $\underline{\text { hs09aa@leeds.ac.uk }}$ 


\begin{abstract}
Rationale, aims and objectives: In Nigeria, a shift from the traditional pharmacists' role of dispensing and compounding of medications began in the 1980s with the introduction of drug information services and unit dose dispensing systems in some hospitals. More than three decades after this, clinical pharmacy practice is still underdeveloped. This study was conducted to explore stakeholders' views on the barriers to the development of clinical pharmacy practice in Nigerian hospitals.
\end{abstract}

Methods: Qualitative, semi-structured interviews were conducted with 44 purposefully sampled Nigerian stakeholders including pharmacists, pharmacy technicians, doctors, policymakers and patient group representatives. Transcribed interviews were entered into the QSR NVivo 10 software and analysed thematically.

Results: Three major themes emerged from the study: pharmacists' professional identity, the structure of pharmacy practice and external barriers. The results revealed an ongoing struggle by Nigerian hospital pharmacists to establish their clinical identities as many non-pharmacy stakeholders viewed pharmacists' roles to be mainly supply-based. Barriers to the development of clinical pharmacy practice identified included pharmacists' lack of confidence, shortage of pharmacy staff, underutilisation of pharmacy technicians, lack of specialisation and clinical career structure, medical dominance and opposition, and lack of policies that support clinical pharmacy practice.

Conclusion: Several years after its introduction, clinical pharmacy practice is yet to be fully developed in Nigerian hospitals. The barriers identified in this study need to be addressed in order for clinical pharmacy practice to flourish.

Keywords: Clinical pharmacy, hospital pharmacy, barriers, Nigeria 


\section{Introduction}

Clinical pharmacy practice which includes direct involvement of pharmacists in patient care has been well established in many developed countries. In the United States and United Kingdom, pharmacists play key roles in patient care including reviewing patients' medications, monitoring of patients' therapy, and participation in prescribing decisions as well as prescribing within collaborative healthcare teams $[1,2]$. The benefits of clinical pharmacy practice have been well established. Many research reports have demonstrated the effectiveness of clinical pharmacists in identifying and resolving drug therapy problems and improving patients' clinical outcomes including a reduction in medication errors, adverse drug events, period of hospitalisation and mortality rates $[1,3]$.

In Nigeria, a shift from the traditional pharmacists' role of dispensing and compounding of medications began in the 1980s with the introduction of drug information services and unit dose dispensing systems in some hospitals [4]. Following these developments, clinical pharmacy courses were made mandatory in the undergraduate pharmacy curriculum in 1990 [5]. More than two decades after this, clinical pharmacy practice is still underdeveloped as it is yet to be institutionalised. In many hospitals, pharmacists' roles are still limited to dispensing of medications and inventory control [6]. For example, a questionnaire survey conducted among 119 respondents including hospital pharmacists in a Nigerian city that assessed the application of a 52 suggested pharmaceutical care practice standards found that only $18.2 \%$

of the respondents were applying such standards in their practice [7]. More recently, a questionnaire survey conducted among 105 pharmacists including those in hospital and community practice in a state in Nigeria revealed that none of the respondents had fully implemented the concept of pharmaceutical care into their practice. Of the 47 hospital pharmacists that participated in the study; only 2 (4.3\%) reported that they participate on ward rounds with other members of the healthcare team [8].

Many studies have identified a number of challenges associated with the development of clinical pharmacy practice in Nigeria. These challenges include opposition from doctors, 
shortage of pharmacy staff and inadequate clinical education [7, 9]. However, these studies were quantitative and therefore, do not provide in-depth understanding of the barriers to clinical pharmacy practice in Nigeria. In addition, the findings they presented were from the perspectives of pharmacists only. Therefore, the studies did not capture the perspectives of the various stakeholders involved in the development of clinical pharmacy practice in Nigeria including doctors and policymakers. This therefore necessitated this study to explore the opinions of different stakeholders on the barriers to the development of clinical pharmacy practice in Nigerian hospitals.

\section{Methods}

\section{Ethics approval}

Ethical approval for this study was obtained from the School of Healthcare Research Ethics Committee, University of Leeds, UK (Ref no: SHREC/RP/344) and the Plateau State Specialist Hospital Health Research Ethics Committee, Nigeria (PSSH/ADM/ETH.CO/2014/0025). All participants were provided an information sheet containing details of the study to enable them make informed decision to participate in the study. A written or oral informed consent was obtained from all research participants.

\section{Study design}

This research employed a qualitative study design using semi-structure interviews to obtain an in-depth understanding of the challenges to clinical pharmacy practice from the perspectives of various stakeholders.

\section{Recruitment of participants}

This study recruited a variety of Nigerian stakeholders including doctors, pharmacists, pharmacy technicians, patient group representatives, and policymakers at the state and Federal Ministry of Health. A purposeful sampling was carried out in order obtain a range of perspectives: mix of stakeholders, practice and geographical settings of pharmacy and medical staff. Generic recruitment emails were sent to 134 prospective participants. These 
were heads of pharmacy and medical departments in some secondary and tertiary hospitals; leaders of professional pharmacy and medical associations; directors at the State and Federal Ministry of Health; and representatives of the Network of People Living with HIV and AIDS in Nigeria.

\section{Data collection}

Semi-structured, in-depth telephone interviews were conducted from April to June, 2014. The interview guide used for the study was developed based on the findings of a research conducted in England which investigated the relationship between pharmacy practice structure and clinical role development [2]. Topic areas covered included participants' perceptions on pharmacists' roles, pharmacists' clinical involvement and contribution to multidisciplinary health team, educational and training provisions for pharmacy staff, career options for pharmacy staff and potential roles for pharmacy staff. The interviews were tailored according to the experience of each stakeholder. All interviews were conducted by AA and digitally recorded following permission from each participant. The interviews conducted in this study were continued until the researcher perceived that data saturation was reached i.e. no new data was generated. Data saturation was perceived to be reached at the $35^{\text {th }}$ interview. However, a total of 44 interviews were conducted. These interviews lasted between 20 and 70 minutes.

\section{Data analysis}

The interviews conducted were fully transcribed. AA checked each transcript against the digital audio recording for accuracy. Transcripts data were entered into the QSR NVivo 10 for appropriate data management. Data were analysed using thematic analysis in the manner outlined by Braun and Clarke [10]. The analysis proceeded in iterative stages of data familiarisation, generation of codes, searching, reviewing, defining and naming of themes. The data were independently coded and analysed by one researcher (AA) but reviewed at different stages by two additional researchers including reviewing and refining the coding structure and 
identified themes. At the final stage of the analysis, identified themes were discussed by the three researchers until a consensus was reached.

\section{Results}

The demographics of the stakeholders interviewed showed a mix of participants in terms of their professional backgrounds, practice settings and geographical locations. The forty four (44) stakeholders interviewed comprised of 15 pharmacists, 9 doctors, 11 policymakers, 6 pharmacy technicians and 3 patient group representatives.

The 11 policymakers interviewed were from the federal (5) and state (6) levels. The 15 pharmacists who participated in this study included 4 academics and 11 hospital pharmacists; while the 9 doctors were 4 consultants, 3 residents, and 2 general practitioners. The pharmacists interviewed had from 5 to 37 years of experience while the doctors had from 10 to 37 years of experience. The majority of the health care professionals interviewed including doctors, pharmacists and pharmacy technicians worked in secondary and tertiary facilities located in urban centres. However, 8 participants were practising in secondary hospitals located in rural centres.

Three major themes emerged from the study: pharmacists' professional identity, the structure of pharmacy practice and external barriers. These themes are represented in Figure 1.

\section{Professional identity}

This theme relates to how various stakeholders characterised pharmacists in relation to who they are and what they do. Overall, pharmacists' identities were largely described by the role they performed. Analysis of the interview data revealed five 'identities' of hospital pharmacists: underutilised professionals, custodians of medicines, dispensers, medicines advisers and clinical practitioners. 
Pharmacists were generally seen by all stakeholders as highly educated professionals whose skills are underutilised within their current scope of practice. Their roles in many hospitals were limited to medicines handling and supply.

"I think the Nigerian pharmacists are highly educated professionals but their roles are greatly limited compared to the vast amount of knowledge and skills acquired." P01, Hospital Pharmacist

"When we were in the medical school, I used to look at pharmacists as learned colleagues, but now in practice, their roles are so few." P34, Resident Doctor

Hence, many stakeholders interviewed including doctors, patient group representatives and policymakers viewed pharmacists as dispensers of medicines.

"A pharmacist is a trained professional that deals with the aspect of drug dispensing in the hospital but I have also found out that they are beginning to counsel patients regarding how the drugs relate to their health circumstances." P38, Patient group representative

"They are the professionals when it comes to drug dispensing." P28, Medical Consultant

Therefore, some doctors interviewed did not see pharmacists' role beyond the dispensary.

These doctors would want pharmacists to further develop their roles in dispensing and other medicine supply functions rather than extending into clinical roles.

"I think it is all about improving on the existing roles they have, I don't think they need to add any more roles to what they are already doing." P28, Medical Consultant

"They should be more involved in compounding a lot of drugs in the hospital. They should also do more by educating patients on the proper way of taking drugs." P29, Resident Doctor

A policymaker with a medical background also holds similar view.

"Dispensing of drugs is not what everybody can do; you need someone who has been trained in that. I want pharmacists to concentrate on that area of specialisation to support the doctors in their practice." P33, Policymaker

This perception held by these doctors showed a great unawareness among doctors of pharmacists' professional roles in patient care. Pharmacists interviewed observed that 
doctors' perception of their roles as being product focussed has been of a barrier to extending their clinical roles.

"The doctors have decided not to know what we can contribute in patient care, they just look at us as drug experts that are just trained to handle drugs like procurement, and dispensing and that is all." P25, Hospital Pharmacist

"This is a bit of a challenge to most of the teaching and specialist hospitals in Nigeria because the medical doctors have refused to come to terms with the fact that the pharmacist has an input especially as regards patients' therapy in the hospital wards." P31, Policymaker with a pharmacy background

In contrast, a few doctors saw pharmacists' role beyond dispensing and would like to have pharmacists contribute to decision making concerning patients' therapy and influence their prescribing as well.

"If I want to prescribe say Haloperidol and while trying to reach that decision with my resident doctors or other consultants during ward rounds, I will also like to have input from the pharmacist saying $X, Y, Z$ pharmacokinetics, why not choose Risperidone,... that kind of contribution, not only doctors' input." P14, Medical Consultant

Furthermore, pharmacists were seen as medicines advisers to both patients and medical doctors. Many doctors reported that hospital pharmacists were resourceful in providing medicine information and advice to them.

"Once in a while, when I need certain clarification on a medicine, I also do call them." P28, Medical Consultant

However, analysis of the interview data showed that the medicine information sought by doctors were mainly product-based. They consist mainly of information related to the formulation, strength and dosage of medicines rather than information on the clinical application of medicines. 


\section{Structure of pharmacy practice}

This theme relates to the organisation and attributes of pharmacy practice in Nigeria. Five subthemes emerged under this theme as seen in Figure 1. Hospital pharmacists were seen as generalists because they do not specialise in clinical areas in their practice.

"The problem with pharmacy practice is that pharmacists are jack of all trades, master of none. But specialisation creates a niche and you become more knowledgeable." P16, Academic Pharmacist

Also, many hospital pharmacists noted that their present career structure does not support them to develop into competent clinical practitioners. The career structure consists of a single route which increasingly becomes managerial as they progressed up the career ladder.

"The present career pathway leads to a managerial function, it does not encourage pharmacists to develop clinically and the promotion from one cadre to another is based on the number of years of experience and not the specialist skills of the pharmacist." P03, Hospital Pharmacists

Hospital pharmacists' confidence to engage in clinical activities at the ward level emerged as a sub-theme. Many participants including doctors and pharmacists observed that a major barrier to pharmacists participating in clinical roles is the lack of confidence shown by pharmacists in clinical settings. This lack of confidence was associated with pharmacists' training. Many participants felt that the current pharmacy undergraduate training programme does not produce practitioners who are confident enough to apply their knowledge in clinical settings.

"They [pharmacists] are not confident enough which again I want to attribute to the training. I think if the training is adequate in that respect, their confidence in participation in such activities like ward rounds will also increase." P14, Medical consultant

"Medical students are trained with patients and they graduate and become residents with patients but that is not the case with pharmacy students. So, there is a need to correct this anomaly with regards to pharmacists' clinical training." P16, Academic pharmacist 
Therefore, some hospital pharmacists felt underprepared for clinical roles. This was obvious from their response to interview questions investigating their clinical involvement in patient care

"You know, pharmaceutical care services are about sound knowledge... We don't want a situation where we join them [doctors] on a ward rounds and the pharmacists are not well prepared." P05, Hospital Pharmacist

The shortage of hospital pharmacists were commonly mentioned by participants as a barrier to clinical pharmacy practice in Nigerian hospitals.

"Of course, inadequate staff is one of the barriers. Definitely when you have very few pharmacists, they may not have time to do other additional roles apart from their dispensing and may be drug distribution." P05, Hospital Pharmacist

Participants associated the shortage of pharmacists to a number of factors. These included inadequate number of pharmacists' training institutions; challenges in recruitment and retention of experienced pharmacists; poor remuneration and migration of pharmacists to other countries for better practice environment and condition of service.

"You know there are a lot of opportunities outside the government for pharmacists. So, very few pharmacists will want to remain with the government like some of us are now. Coupled with the condition of service and remuneration in the public sector, most pharmacists will prefer to take up non-governmental organisation work and other private endeavours." P35, Policymaker

In addition to the shortage of hospital pharmacists, pharmacy technicians are usually underutilised.

"They will hardly allow you manage the dispensary for them to go to the wards because they believe that there are certain things that they feel they should be the ones to control." P41, Pharmacy Technician However, in order to free up pharmacists' time to undertake clinical roles, pharmacy technicians would be needed to fulfil some traditional roles of pharmacists including dispensing. 
I believe that dispensing should be things that technicians should be doing. I feel the pharmacist should be more involved in direct patient care. You know, go to the ward and provide services to patients in the ward. But right now pharmacists are involved in the packing of drugs, counting of drugs and all that I believe those things should be left for the technicians, which will now allow a pharmacist to have more time to render pharmaceutical care services to patients. P05, Hospital Pharmacist

Nevertheless, there was a perception that the extension of some pharmacists' traditional medicines supply roles including dispensing, to pharmacy technicians represents a threat to pharmacists' professional status.

"They are afraid that if we are giving the chance to work in this way [managing the dispensary], we will overtake their duties as pharmacists" P46 Pharmacy Technician

This was perceived as a threat because many pharmacists considered the control they have over medicine supply as a major reason for their professionalism.

\section{External barriers}

Hospital pharmacists observed that the participation of pharmacists in patient care on hospital wards has been resisted by medical doctors. They reported that medical doctors believe that the care of patients in the hospitals is solely under their responsibility and allowing other healthcare professionals to be directly involved in patient care would undermine their control over it.

"The products of the West African Postgraduate College of Pharmacists are finding it difficult to actually practice because the doctors feel that they have gone to read clinical pharmacy to take over the clinics; that is their perception which in the real sense is not." P25, Hospital Pharmacist

A policymaker with a medical background also pointed this out as a barrier to collaborative practice in patient care in Nigeria.

"You know, the doctor is naturally a selfish person especially when it comes to patient care. The doctor wants to take the credit at the end of the day, I diagnose it, I treated it, I did this... So, there is this feeling that if you work collaboratively, who takes the credit at the end of the day?" P34, Policymaker 
Hence, collaborative working relationship between pharmacists and doctors is lacking as clearly indicated by a participant below.

"Interdisciplinary collaboration does not exist currently in Nigeria. They [referring to doctors and pharmacists] fight for positions instead of fighting for excellence in patient care. The patients greatly suffer because there is no collaboration in the system." P30, Policymakers \& medical doctor

Participants also spoke of medical control over the policy and administrative structures of the health system at the national, state and institutional levels. This was seen as a barrier to policy development that will support clinical pharmacy practice. Many non-medical stakeholders especially pharmacists argued that the medical profession has majority of membership in most policy making bodies in the health sector. Therefore any policy that does not favour them or tend to challenge their existing control of the health sector is usually resisted by doctors.

"I have had cause to present memoranda during XXX council. I can say categorically the attack, if I may use the word, or the response from the medical practitioners that form the bulk of the members of the council have been bad. Simply because, they look at pharmacists as trying to go into their own "preserved area'." P31, Policymaker

Overall, many pharmacists maintained that achieving professional autonomy in terms of pharmacists' ability to control its administrative structures, negotiate its roles and remuneration is essential for clinical role extension.

"Pharmacists don't have enough political strength to make clinical pharmacy practice a reality in Nigeria. I think more pharmacists should delve into politics in order for us to canvass for our own rights within the health sector." P13, Hospital Pharmacist

These participants believed that having more pharmacists in government will facilitate pharmacists' ability to negotiate their roles.

\section{Discussion}

This study revealed how different stakeholders socially perceive and construct the role of hospital pharmacists in Nigeria. In addition, it demonstrates how the perceived professional identity of hospital pharmacists could influence participants' support for pharmacists' extended 
roles. For example, many doctors who viewed hospital pharmacists as dispensers of medicines did not see pharmacists' roles beyond medicine supply functions. Therefore, they were unsupportive of pharmacists' direct involvement in patient care including pharmacists' participation in therapeutic decision making and monitoring of patient therapy. These doctors would prefer pharmacists to concentrate on medicine supply and advisory roles.

The present study also revealed that clinical pharmacy practice is still underdeveloped in Nigerian hospitals and many factors were responsible for this. These factors were predominantly linked to the structure of pharmacy including pharmacists' lack of confidence which was associated with their training, lack of specialisation in practice, a career structure that does not support clinical development, shortage of pharmacists and underutilisation of pharmacy technicians.

The present study has indicated that the undergraduate pharmacy programme in Nigeria does not produce confident pharmacists who are able to apply their knowledge in clinical settings. Even though the curriculum of the undergraduate pharmacy programme in Nigeria has changed significantly $[11,12]$, the clinical components of the present curriculum in many pharmacy institutions is insufficient for clinical pharmacy practice. In addition, experiential learning through clinical rotations which will potentially enhance pharmacists' clinical skills and confidence in clinical settings is inadequate [11]. In the undergraduate training curriculum of many pharmacy institutions in Nigeria, clinical pharmacy rotations are usually carried out in the final year of the undergraduate programme. These clinical rotations only account for about $10 \%$ of the final year curriculum [13]. Furthermore, when students are involved in clinical rotations, they are usually conducted in settings where clinical pharmacy services are yet to be fully developed which limits students' practice-based learning experience [11]. Therefore, to enhance pharmacists' confidence, the current training curriculum needs to incorporate more clinical contents with practice based learning conducted at sites where clinical pharmacy services are offered. However, finding appropriate training sites will continue to be a challenge as long as pharmacists' roles in many hospital settings remain underdeveloped. 
Another factor that could account for the lack of confidence seen among pharmacists in clinical settings could be the absence of a national framework to evaluate the skills and competencies of those entering into the profession. Unlike in many advanced countries including the UK, there is currently no pre-registration assessment for pharmacists [14]. New graduates enter into the profession after a year of pre-registration training in an accredited centre. At the end of the training programme, these graduates are issued a 'certificate of experience' as evidence of experience with a registered pharmacist which is a pre-requisite for application to register as a pharmacist in Nigeria [14]. Even though the pre-registration training centres are accredited by the Pharmacists Council of Nigeria (PCN), the potential for variation in training among the various training centres is high. Therefore a national pre-registration assessment is essential to ensure that pharmacists achieve minimum competences standard before entering into the profession.

Furthermore, the research evidence from England has shown that practice-based postgraduate clinical pharmacy programmes are essential in enhancing pharmacists' clinical skills and confidence in clinical settings [2]. Many researchers have argued that these programmes provide a specialised level of training that cannot be achieved within the undergraduate pharmacy programme [15-17]. This is because the undergraduate pharmacy programme prepares pharmacists for practice in diverse kind of settings including non-patient care settings. Therefore, it cannot achieve the level of depth needed for independent practice in patient care settings [15]. Hence many countries including the UK and US have taken up post-registration clinical pharmacy training in order to prepare pharmacists' for specialised roles in clinical settings [16].

In Nigeria, masters of clinical pharmacy programmes are available in some Universities [12]. However, these programmes are largely theoretical with little practice based learning. More recently, the West African Postgraduate College of Pharmacists (WAPCP) has played a significant role in the postgraduate training of pharmacists in clinical pharmacy. WAPCP was established by the West African Pharmaceutical Federation in 1991 to prepare pharmacists in 
the West African region including Nigeria for expanded roles [18]. The clinical pharmacy programme of the WAPCP is a distance learning programme of 4 years duration incorporating two cycles of update lectures annually [19]. In 2014, a residency component was introduced into the programme with some of the graduates of the programme serving as preceptors at practice site [20]. However, the effectiveness of the residency programme would largely depend on the development of clinical pharmacy services at the selected practice sites of the residents.

Another important barrier to the development of clinical pharmacy practice in Nigeria is the shortage of pharmacists. Other research reports from Nigeria have also highlighted the shortage of pharmacists as a barrier to providing pharmaceutical care $[7,9]$. This is unsurprising considering the ratio of pharmacists to the population as there are approximately 17,000 pharmacists in Nigeria which represents about 10 pharmacists to 100,000 people [21].

The classical response to pharmacists shortages is to increase the production of new pharmacists by increasing the number of pharmacists training institutions or student intake; or to increase the utilisation of pharmacists' support staff in order to free up pharmacists for advanced roles [22]. Both strategies have been recommended and implemented in response to pharmacists shortages in many advanced countries including Australia, UK and the USA [23]. However, increasing the production of new pharmacists does not present a short term feasible option for Nigeria in view of the constraints associated with it including inadequate academic staff. In addition, it will take a minimum of five years (following initial plan to increase pharmacists' numbers) to produce new pharmacy graduates since pharmacy programmes last for a minimum of 5 years in Nigeria. Therefore, increasing the utilisation of the current pharmacy technicians' workforce represents an immediate solution with long term benefits for Nigeria.

Available international evidence supports the pharmacy technicians' role in dispensing [2, 24]. For example, a prospective study conducted in three large hospitals in Washington State 
shows no difference between pharmacists' and trained pharmacy technicians' accuracy checks of dispensed medicines [24]. Generally, dispensing of medicines in many Nigerian hospitals usually consists of a series of steps. This process begins with validation of prescriber's and patient's information following the receipt of a prescription. After which, the prescription is clinically validated. This is then followed by the selection, assembling and labelling and entering of labelled medicine items into the register. The final phase of the dispensing process involves accuracy checking, packaging and delivery of the medicines to patients. Patient counselling is usually done at the point of the delivery. The active involvement of pharmacists in all of these stages account for why they are usually tied to the dispensary. Thus, utilising pharmacy technicians in the physical aspects of dispensing following the clinical check of prescription including selection, assembling, labelling and accuracy checks of final dispensed medicines would free hospital pharmacists' up to do more clinical roles. However, for technicians' roles to be considerably expanded to include accuracy checks of final dispensed medicines, additional training and accreditation as currently done in the UK would be needed.

The need for a career pathway for clinical pharmacists and their specialisation in practice were underscored by many participants including pharmacists. Auta et al. [2], have argued that a clinical career route has the potential to make pharmacists aspirational and motivate them to develop clinical competencies that are needed for advanced practice in order to progress through the career ladder. In addition, these authors maintained that it could promote the retention of experienced clinical staff who desire to use their expertise to enhance patient care rather than move into managerial roles where their clinical expertise would be untapped.

Specialisation in practice is easily achievable if clinical career pathways and practice-based post-registration trainings such as residency training programmes are available $[2,15,25,26]$. The residency components of the WAPCP programme mentioned earlier can be used to facilitate specialisation within clinical areas. However, the specialisation of pharmacists' into different clinical areas would take pharmacists away from general functions. This would 
potentially impact negatively on the number of pharmacists that are available for generalist roles and worsen the present shortage of pharmacists in Nigerian hospitals. Therefore, a more flexible and practical approach to specialisation is needed in Nigeria. In the interim, the training of advanced generalist practitioners with additional specialist skills should be explored. This would allow pharmacists to be useful in both generalist and specialist areas of their interest.

Other challenges to clinical pharmacy practice in Nigerian hospitals identified were medical opposition and poor professional working relationships between doctors and pharmacists. In Nigerian hospitals, doctors control the boundaries of the professional work of other healthcare professionals because they are perceived to have the prime responsibility of patient care. Therefore, they define the role played by other healthcare professionals in patient care. Hence, pharmacists' direct involvement in patient care has always been viewed by Nigerian doctors as a an encroachment into their professional boundary and has often times been resisted [4].

Zillich et al. [27], suggested that collaborative working relationship with doctors can be initiated by establishing communication with doctors through the development of services that improve doctors' care of the patient [27]. This will involve identifying a gap in doctors' practice where pharmacists can fill in positively. Furthermore, many authors have argued that doctors with positive experience of clinical pharmacists are usually supportive of clinical pharmacy practice $[28,29]$. Therefore, Nigerian pharmacists must seek to identify areas where they can make positive contributions that will enhance doctors' care of the patient and ultimately result in better communication and closer working relationship between them and doctors.

Also, working in collaboration with other healthcare professionals requires the knowledge of each other's roles [15]. Many authors have argued that collaboration between healthcare professionals increases as they become aware of each other's knowledge and skills [30, 31]. Therefore, inter-professional education has been suggested as a means of promoting interprofessional understanding among healthcare professionals [30]. In Nigeria, pharmacy and medical students are trained in isolation from one another (uni-professional education). This 
form of learning may account for the stereotype views held by doctors concerning pharmacists' role in patient care in this study. Therefore, joint learning sessions within undergraduate programmes would help to promote understanding of the roles of both professionals in patient care. Other measures that could foster relationships between pharmacists and doctors include providing opportunities for pre-registration pharmacists to work with doctors during internship training; organising joint continuing education events or clinical meetings; and having policies/legislations that would enhance collaborative practice [32].

\section{Conclusion}

The present study revealed that clinical pharmacy practice is still underdeveloped in Nigerian hospitals and many factors were responsible for this. These factors were predominantly linked to the structure of pharmacy. They include limited clinical education and training, lack of specialisation in practice, a career structure that does not support clinical development, shortage of pharmacists and underutilisation of pharmacy technicians. Other barriers identified were medical opposition, poor relationship between pharmacists and medical doctors and inadequate policies to support the development of clinical pharmacy practice. These barriers would need to be addressed in order for clinical pharmacy practice to grow in Nigeria.

\section{Acknowledgement}

The authors are grateful to all the participants of this study.

\section{Conflict of interest}

The authors do not have any financial or conflict of interest to declare.

\section{References:}

1. Bond, C. \& Raehl, C. L. (2007). Clinical pharmacy services, pharmacy staffing, and hospital mortality rates. Pharmacotherapy, 27(4), 481-493.

2. Auta, A., Maz, J. \& Strickland-Hodge, B. (2015). Perceived facilitators to change in hospital pharmacy practice in England. International Journal of Clinical Pharmacy, DOI: 10.1007/s11096-015-0153-9.

3. Bond, C. \& Raehl, C. L. (2006). Clinical pharmacy services, pharmacy staffing, and adverse drug reactions in United States hospitals. Pharmacotherapy, 26(6), 735-747. 
4. Erah, P. O. (2003). The changing roles of pharmacists in hospital and community practice in Nigeria. Tropical Journal of Pharmaceutical Research, 2(2), 195-196.

5. Brown, A. A. \& J.J. Ogun, J. J. (1998). Clinical pharmacy practice development in Nigeria: a historical account. Lagos: Sedoten Ventures.

6. Auta, A., Fredrick, N.C, David, S., Banwat, S.B. \& Adeniyi, M.A. (2014). Patients' views on their consultation experience in community pharmacies and the potential prescribing role for pharmacists in Nigeria. Journal of Pharmaceutical Health Services Research, 5, 233-236.

7. Erah, P.O. \& Nwazuoke, J.C. (2002). Identification of standards for pharmaceutical care in Benin City. Tropical Journal of Pharmaceutical Research, 1(2), 55-66.

8. Suleiman, I.A. \& Onaneye, O. (2011). Pharmaceutical care implementation: A survey of attitude, perception and practice of pharmacists in Ogun State, South-Western Nigeria. International Journal of Health Research, 4(2), 91-97.

9. Oparah, A.C. \& Eferakeya, A.E. (2005). Attitudes of Nigerian pharmacists towards pharmaceutical care. Pharmacy World \& Science, 27(3), 208-214.

10. Braun, V. \& Clarke, V. (2006). Using thematic analysis in psychology. Qualitative Research in Psychology, 3(2), 77-101.

11. Udeogaranya, P.O., Ukwe, C.V., \& Ekwunife., O.I. (2009). Assessment of attitudes of University of Nigeria pharmacy students toward pharmaceutical care. Pharmacy Practice (Internet), 7(3), 145-149.

12. Ogaji, J.I. \& Ojabo, C.E. (2014). Pharmacy education in Nigeria: The journey so far. Archives of Pharmacy Practice, 5(2), 47-60.

13. National Universities Commission (2007). Benchmark minimum academic standards for undergraduate programmes in Nigerian universities: Pharmaceutical sciences. Abuja: National Universities Commission.

14. Pharmacists Council of Nigeria (2014). Application for Registration as a Pharmacist (Form C). Available at: http://pcn.gov.ng/Form.htm

15. Jungnickel, P.W., Kelley, K.W., Hammer, D.P., Haines, S.T. \& Marlowe, K.F. (2009). Addressing competencies for the future in the professional curriculum. American Journal of Pharmaceutical Education, 2009, 73(8), 156.

16. Leiker, L.L., Mehta, B.H., Pruchnicki, M.C. \& Pedersen, C. A. (2009). Assessment of pharmacy faculty members' opinions regarding required postgraduate pharmacy residencies. American Journal of Health-System Pharmacy, 66(4), 378-388.

17. Murphy, J.E., Nappi, J. M., Bosso, J. A., Saseen, J.J., Hemstreet, B. A., Halloran, M. A., Spinler, S. A., Welty, T. E., Dobesh, P. P. \& Chan, L. (2006). American College of Clinical Pharmacy's vision of the future: postgraduate pharmacy residency training as a prerequisite for direct patient care practice. Pharmacotherapy, 26(5), 722-733. 
18. West African Postgraduate College of Pharmacists (2013). West African Postgraduate College of Pharmacists: Historical Background. Available from: http://www.wapharm.org/pm/webpages.php?id=1.

19. West African Postgraduate College of Pharmacists (2012). Regulations for diploma programs. Available from: http://www.wapcp.info/about/regulations-diploma-programs

20. West African Postgraduate College of Pharmacists (2014). Residency guidelines. Available from: http://www.wapharm.org/pm/login.php

21. Pharmacists Council of Nigeria (2014). Full registration list of Pharmacists as at 2nd October, 2014. Available from: http://pcn.gov.ng/WEB\%20full\%20registration\%20list.pdf

22. Cooksey, J.A., Knapp, K.K., Walton, S. M. \& Cultice, J. M (2002). Challenges to the pharmacist profession from escalating pharmaceutical demand. Health Affairs, 21(5), 182-188.

23. Anderson, C., Bates, I., Beck, D., Brock, T. P., Futter, B., Mercer, H., Rouse, M., Whitmarsh, S., Wuliji, T. \& Yonemura, A. (2009). The WHO UNESCO FIP pharmacy education taskforce. Human Resources for Health, 7, 45.

24. Ness, J.E., Sullivan, S.D. \& Stergachis A (1994). Accuracy of technicians and pharmacists in identifying dispensing errors. American Journal of Health-System Pharmacy, 51(3), 354-357.

25. Storey, M., White, S. \& Weber, R. (2011). Practice Models: What Are Other Professions Doing? Hospital Pharmacy, 46(9), 709-718.

26. Bright, D.R., Adams, A. J., Black, C. D. \& Powers, M. F. (2010). The mandatory residency dilemma: parallels to historical transitions in pharmacy education. Annals of Pharmacotherapy, 44(11), 1793-1799.

27. Zillich, A.J., McDonough, R. P., Carter, B. L. \& Doucette, W. R. (2004). Influential Characteristics of Physician/Pharmacist Collaborative Relationships. Annals of Pharmacotherapy, 38(5), 764-770.

28. Adamcik, B.A., Ransford, H. E., Oppenheimer, P. R., Brown, J. F., Eagan, P. A. \& Weissman, F. G. (1986). New clinical roles for pharmacists: a study of role expansion. Social Science \& Medicine, 23(11), 1187-200.

29. Ritchey, F.J. \& Raney, M.R. (1981). Medical role-task boundary maintenance: physicians' opinions on clinical pharmacy. Medical Care, 19(1), 90-103.

30. Zwarenstein, M., Goldman, J.\& Reeves, S. (2009). Interprofessional collaboration: effects of practice-based interventions on professional practice and healthcare outcomes. Cochrane Database of Systematic Reviews, 8(3), CD000072. 
31. O'Daniel, M. \& Rosenstein, A.H. (2008). Professional communication and team collaboration. In patient safety and quality: an evidence-based handbook for Nurses (ed R.G. Hughes) Rockville (MD): Agency for Healthcare Research and Quality

32. Van, C., Costa, D., Abbott, P., Mitchell, B. \& Krass, I. (2012) Community pharmacist attitudes towards collaboration with general practitioners: development and validation of a measure and a model. BMC Health Services Research, 12(1), 320.

Figure

\section{Professional Identity}

- Underutilised professionals

- Custodians of medicines

- Dispensers

- clinical practitioners

- Medicine advisers

\section{Structure of} pharmacy practice

- Lack of confidence

- Lack of specilisation in practice

- Lack of a clinical career pathway

- Shortage of pharmacists

- Underutilisation of pharmacy technicians

\section{External barriers}

- Medical dominance and opposition

- Poor working relationship with doctors

- Lack of policy support

Figure 1: Themes and subthemes identified 